\title{
Evolution of ultra-massive stars
}

\section{Lev R. Yungelson}

Institute of Astronomy, Pyatnitskaya 48, 119017 Moscow, Russia email: lry@inasan.ru

Abstract. We discuss the evolution of ultra-massive stars.

Keywords. stars: evolution, stars: mass-loss

We have calculated evolution of $M_{0}=(60-1001) \mathrm{M}_{\odot}$ non-rotating solar-metallicity stars from main-sequence through core helium-burning stage. The value of $1001 \mathrm{M}_{\odot}$ is the upper mass limit for ZAMS of solar-metallicity stars. Assuming that $\Gamma$-instability is responsible for mass loss we applied an ad hoc mass-loss law $\dot{M}=\frac{L}{v_{\infty} c} \frac{1}{\left(1-L / L_{\mathrm{Edd}}\right)^{\alpha}}$. Here $L_{\mathrm{Edd}}$ is the Eddington luminosity in the outermost mesh point of the model. The value of $\alpha=0.25$ was chosen such that stars with mass below $120 \mathrm{M}_{\odot}$ spend less that $1 \%$ of their lifetime to the right of Humphreys-Davidson limit in the HR-diagram. Results of computations are relevant to the evolution of the most massive stars in the Galactic Center clusters or for (still hypothetical) products of runaway stellar collisions in young dense clusters that are thought to be progenitors of black hole accretors in some ultraluminous X-ray sources.

Mass-loss rates both for hydrogen- and helium-rich stars that follow from above given expression for $\dot{M}$ are consistent with the limits implied for line-driven winds and with $\dot{M}$ found for the most massive stars, e.g., in Arches, Quintuplet, He I-clusters in the Galactic Center or in $\mathrm{R} 136$.

The end-products of the evolution of ultra massive stars have oxygen-neon cores with minor admixture of carbon and thin helium-carbon envelopes $\left(Y_{s} \approx 0.2-0.4\right)$. Initial-final mass relation obtained in calculations and the fate of stellar remnants (as inferred from model calculations for helium stars) are shown in the Figure. These results suggest that runaway stellar collisions hardly can produce black holes with masses $\gtrsim 100 \mathrm{M}_{\odot}$.

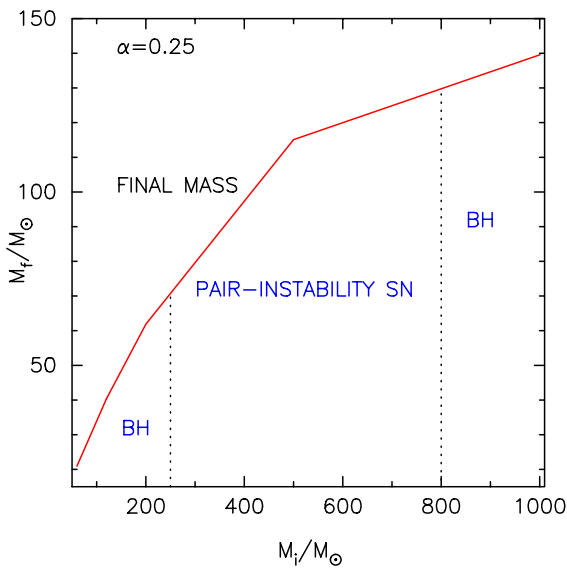

Figure 1. Relation between initial and final masses of stars and the nature of stellar remnants. 